\title{
Evaluation of Chitosan Modified Glass Ionomer Cement for Microleakage under Spectrophotometer - An In-Vitro Study
}

\author{
Arjun Sajjeev ${ }^{1}$, Ashwini Tumkur Shivkumar², Sowmya Halasabalu Kalgeri ${ }^{3}$ \\ 1, 2,3 Department of Conservative Dentistry and Endodontics, JSS Dental \\ College \& Hospital, JSSAHER Mysore, Karnataka, India.
}

\section{ABSTRACT}

\section{BACKGROUND}

Marginal integrity of glass ionomer as a restorative material is an important factor for the longevity of the restoration. Class $V$ and cervical abrasions are the most critical and challenging lesions for restorations. The choice of material for restoring class $\mathrm{V}$ and cervical abrasions is glass ionomer cement (GIC). Sensitivity to moisture contact during the early setting stages is the drawback of GIC. To overcome the drawback, modifications of glass ionomer cement were made by the addition of chitosan. Chitosan $(\mathrm{CH})$ is a natural linear polysaccharide obtained partially and fully by deacetylated chitin compounds, which are found in crab and shrimp shells, with properties like nontoxicity, biodegradability, bioadhesive, biocompatibility, and biorenewabilty which has led to its use in various fields. Thus, this study intended to evaluate the microleakage of conventional glass ionomer cement and chitosan modified glass ionomer cement using a spectrophotometer.

\section{METHODS}

60 teeth extracted for orthodontic propose were selected for the study and randomly divided into two groups, class $\mathrm{V}$ cavities were prepared on the buccal surface and samples were restored with conventional glass ionomer cement and chitosan modified glass ionomer cement respectively, teeth were immersed in $0.5 \%$ methylene blue for 24 hours and assessed using a spectrophotometer.

\section{RESULTS}

The data were analysed using the Unpaired T - test, and with statistical package for social sciences (SPSS) for Windows, version 25.0 (IBM Corp., Armonk, N.Y., USA). The confidence interval was set at $95 \%$ and values of $\mathrm{P}<0.05$ were interpreted as statistically significant.

\section{CONCLUSIONS}

The study concluded that the addition of chitosan improves the mechanical properties of conventional glass ionomer cement, and a spectrophotometer can be used as a better evaluation tool in assessing microleakage.

\section{KEY WORDS}

Chitosan Modified GIC, Glass Ionomer Cement, Microleakage, Spectrophotometer
Corresponding Author: Dr. Ashwini Tumkur shivkumar, Associate Professor, Department of Conservative Dentistry and Endodontics, JSS Dental College \& Hospital, JSSAHER, Mysore - 570015, Karnataka, India.

E-mail: drashts2000@gmail.com

DOI: 10.14260/jemds/2021/565

How to Cite This Article:

Sajjeev A, Shivkumar AT, Kalgeri SH. Evaluation of chitosan modified glass ionomer cement for microleakage under spectrophotometer - an in-vitro study. J Evolution Med Dent Sci 2021;10(33):27692772, DOI: $10.14260 /$ jemds/2021/565

Submission 08-04-2021,

Peer Review 11-06-2021,

Acceptance 18-06-2021,

Published 16-08-2021.

Copyright (C) 2021 Arjun Sajjeev et al. This is an open access article distributed under Creative Commons Attribution License [Attribution 4.0 International (CC BY 4.0)] 


\section{BACKGROUND}

Over the years, aesthetics in dentistry has become prime consideration, and this has led to the development of several improvements in restorative materials. The concern was the clinical performance of these materials regarding their marginal sealing and durability especially when the cavity margin lies on the cementum region, increased clinical problems has led to the development of modified aesthetic restorations

Glass ionomer cement (GIC) was introduced by Wilson and Kent, which has unique properties and is beneficial as an adhesive restorative material. ${ }^{1}$ It consists of a basic glass powder, a calcium (or strontium) alumina fluorosilicate and a water-soluble acidic polymer, such as poly (acrylic acid). Since it was introduced, researchers are keen on improving the properties of glass ionomer cement. ${ }^{1}$ Their main advantages are relative ease of use, bonding potential to enamel and dentin, and fluoride ion release. The disadvantages of glass ionomer cement are sensitivity to moisture contact and desiccation during the early setting stages. Cervical lesions such as class $\mathrm{V}$ and cervical abrasions have been tough to restore. The complex margins of Class $V$ cavities, which fall on enamel as well as on dentin, present a challenging situation for the dentist. The challenge associated with restoring of these cavities is preventing leakage at the gingival margin. ${ }^{2}$ The most common problem of restorative dentistry is the failure to obtain a complete bond with enamel and dentin. Despite good chemical adhesion glass ionomer cements show some micro leakage at the margins of restorations. To overcome this disadvantage, chitosan, a natural linear polysaccharide was added to improve the properties of glass ionomer cement

Chitosan $(\mathrm{CH})$ is a natural linear polysaccharide obtained partially and fully by deacetylated chitin compounds, ${ }^{3}$ which are found in crab and shrimp shells. ${ }^{4}$ Chitin, abundant carbohydrates are commonly obtained from crustacean exoskeletons. ${ }^{4,5}$ Its characteristic properties are nontoxicity, biodegradability, bioadhesive, biocompatibility, and biorenewabilty which has led to its use in various fields $s^{6,7}$ such as drug delivery, tissue engineering, and healthcare products. ${ }^{8}$ Chitosan is widely considered to be a non-toxic, compatible, biological polymer and therefore allowed for dietary applications. ${ }^{9}$ Chitosan has been widely used in dentistry including augmentation of edentulous ridge, ${ }^{10}$ osteogenesis, ${ }^{11}$ increasing salivary secretion, ${ }^{12}$ as a guided bone regeneration tool ${ }^{13}$ and to improve wound healing. ${ }^{14}$

Chitosan has been introduced in dentistry because of its stable crystalline structure which is insoluble in alkaline $\mathrm{pH}$, which results in a strong hydrogen bond. ${ }^{15}$ Hence the conventional GIC was added with chitosan to evaluate micro leakage in-vitro using a spectrophotometer

Microleakage evaluation is an ideal assessment to determine the success of any restorative material. Micro leakage can be explained as the movement of bacteria, body fluids, molecules, or ions, and even air between the restorative materials and the prepared cavity walls. ${ }^{2}$ The ideal requirement of a good restorative material is bonding to the tooth structure and failure to achieve this leads to microleakage and failure of the restoration. This eventually leads to, marginal breakdown, postoperative hypersensitivity, and recurrent caries.

\section{METHODS}

This in-vitro comparative study was carried out for over 6 months from May to October 2019 in the Department of Conservative Dentistry and Endodontics, JSS dental college and Hospital, JSSAHER, Mysore.

The research protocol was approved by the JSSDCH Institutional ethical committee, Mysore. The sample size of the study was calculated from the standard deviation, significance, and power from the previous studies. A total of 60 caries - free premolars extracted for orthodontic purposes were selected and were systematically randomised into two groups. Group A restored with conventional glass ionomer cement (GC Fuji II, GC cooperation, Tokyo, Japan) and Group B with chitosan modified glass ionomer cement.

\section{Preparation of Chitosan Modified Glass Ionomer Cement}

About $1.8 \mathrm{ml}$ of glacial acetic acid was made up to $100 \mathrm{ml}$ with distilled water in $100 \mathrm{ml}$ standard flask to get $0.3 \mathrm{~N}$ acetic acid. About $20 \mathrm{mg}$ of chitosan, was weighed independently and broken down in $0.3 \mathrm{~N}$ acetic acid and made up to $100 \mathrm{ml}$ with some acetic acid in a $100 \mathrm{ml}$ standard flask to get $0.2 \mathrm{mg} / \mathrm{ml}$ chitosan solution. Then $0.1 \mathrm{ml}$ of $0.2 \mathrm{mg} / \mathrm{ml}$ of chitosan solution was added to $0.9 \mathrm{ml}$ of glass ionomer cement liquid to get $10 \% \mathrm{v} / \mathrm{v}$ chitosan modified glass ionomer solution.

\section{Specimen Preparation}

Class V cavities with the dimension $1.5 \mathrm{~mm} \times 1.5 \mathrm{~mm} \times 1.5 \mathrm{~mm}$ were prepared on the buccal surface of premolar teeth and each cavity was standardized with a Williams graduated probe, restored with respective cement. (Fig. 1)

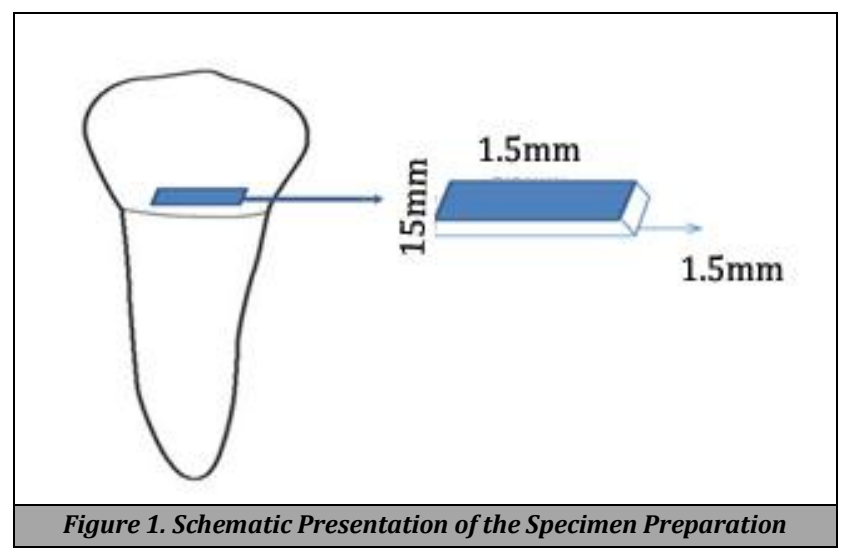

\section{Thermo Cycling and Dye Penetration}

An entire surface of the restoration was isolated with adhesive tape; two coats of nail varnish were applied to leave a $1.5 \mathrm{~mm}$ gap around the restoration. Each group was then individually placed in a separate mesh bag. Thermo cycling was carried out for 500 cycles at a temperature of $5^{\circ} \mathrm{C} \pm 2^{\circ} \mathrm{C}$ and $55^{\circ} \mathrm{C} \pm 2{ }^{\circ}$ $\mathrm{C}$ for each group for 30 seconds and 15 seconds respectively. Upon thermocycling, the apex of the teeth was covered with modelling wax to avoid unwanted dye penetration. Then teeth were individually kept at $1.3 \mathrm{ml}$ of $0.5 \%$ methylene blue at room temperature for 24 hours, and all teeth were removed 
from methylene blue and each tooth was thoroughly rinsed in running water. Nail polish was removed with a blade and surface dye was removed with Sof-Lex disks.

\section{Microleakage Quantification}

Experimental teeth were individually immersed in $2 \mathrm{ml}$ of 65 $\%$ nitric acid in a test tube. After 48 hours teeth placed in nitric acid solutions were diluted by $1 \mathrm{ml}$ distilled water. Then the solutions were centrifuged at $4000 \mathrm{rpm}$ for 20 minutes and the supernatant obtained was transferred to a cuvette and was placed in a spectrophotometer and the absorbance reading was determined at 598 nanometres.

\section{Statistical Analysis}

An unpaired $t$ - test was applied to compare the dye concentration of Group A and Group B. The data were analysed with Statistical Package for Social Sciences (SPSS) for Windows, version 25.0 (IBM Corp., Armonk, N.Y., USA). The confidence interval was set at $95 \%$ and values of $\mathrm{P}<0.05$ were interpreted as statistically significant.

\section{RESULTS}

Both the samples Group A restored with conventional GIC and Group B restored with chitosan modified GIC were assessed for microleakage using a spectrophotometer.

\section{Interpretation}

Intergroup Comparison

Dye concentration of group A conventional glass ionomer cement was $0.776 \pm 0.331$ and group B chitosan modified glass ionomer cement was $0.516 \pm 0.300$ respectively, which showed statistically significant difference with $\mathrm{P}-$ value $=$ $0.002, \mathrm{t}-$ value $=-3.18$. $($ Graph 1$)$. The result showed that chitosan modified glass ionomer cement (Group B) had less microleakage than conventional glass ionomer cement (Group A).

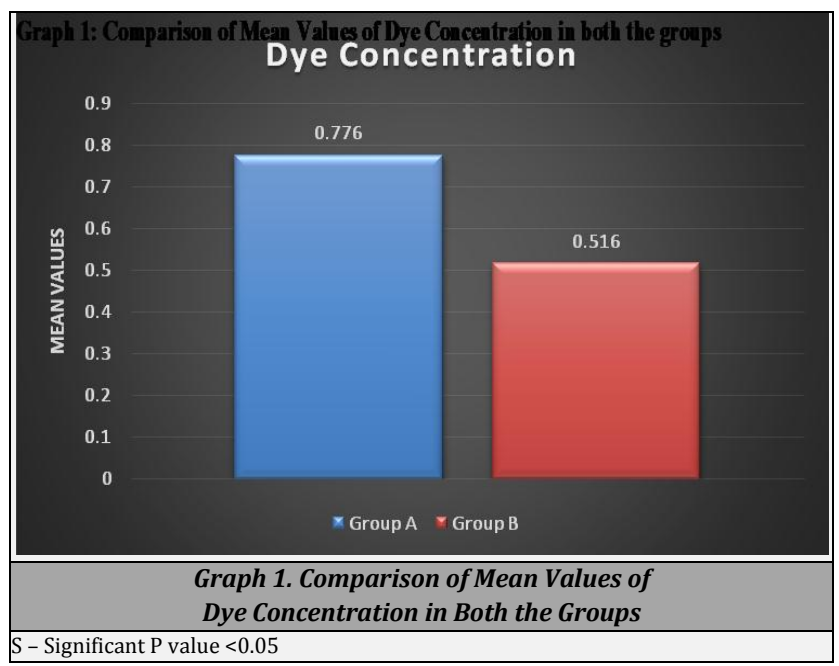

\section{DISCUSSION}

The chemical adhesion of restorative materials to dental tissue is the ideal requirement of a restorative material, they should also have similar physical characteristics of dental tissues, and should be stable in the oral environment. ${ }^{2}$ However, the earlier conventional glass ionomer cements were satisfying the above mentioned requisites to an extent but they also showed limitations such as low resistance to breaking and abrasion, as well as their sensitivity to dryness and moisture. To reduce their susceptibility to moisture, abrasion resistance and stiffness were increased.

Chitosan was selected as a chemical additive to modify and improve the properties of conventional glass ionomer. Accordingly, the potential applications of chitosan in preventive and restorative dentistry are intensively investigated for its possible clinical applications. ${ }^{16}$ The result of this study shows that the incorporation of chitosan $10 \%$ (V / V) decrease the microleakage of conventional glass ionomer cement. Microleakage is the leading cause for the failure of bonded restorative material; microleakage assays provide useful information on the performance of restorative materials. ${ }^{17}$

There are various methods to detect microleakage, that include the use of dyes, chemical tracers, and radioactive tracers; scanning electron microscopy, neutron activation analysis, and fluid filtration. ${ }^{2}$ Many studies have been performed using methylene blue, India ink, basic fuchsin, crystal violet, as well as fluorescein. In the present study, we choose to use $0.5 \%$ methylene blue dye as it is ease to manipulate, convenient, and also has low molecular weight of the dye. The dye adds to an easy visualization of the sample cavity and excellent contrast with the surrounding environment.

Thermocycling is a method most commonly used in research, basically while testing the performance of adhesive materials in conditions which mimic intraoral temperature variations by subjecting the restorations to temperature extremes comparable with the oral environment.

A spectrophotometer is an instrument that helps in physical analysis and provides spectral analysis. Hence it is used to calculate the percentage of dye penetration, which eliminated possible human bias while evaluating microleakage. ${ }^{18}$ In the present study group B (chitosan modified glass ionomer cement) shows less microleakage compared to group A (conventional glass ionomer cement) because of better chemical reaction, adhesion, water sorption, and bond strength of chitosan modified glass ionomer cement.

$\mathrm{CH}$ was used with acetic acid mixed with polyacrylic acid liquid. $\mathrm{CH}$ can be considered a strong material as it contains free amino groups (NH3+) when dissolved with polyacrylic acid. ${ }^{19}$ The chemical reaction occurs between the amino ($\mathrm{NH} 2)$ group of $\mathrm{CHs}$ and the functional group $(\mathrm{OH}$ group and $\mathrm{C}$ $=0$ group) of the conventional glass ionomer cement. Since $\mathrm{CH}$ has hydroxyl and acetamide groups, hydrogen bonding occurs between hydroxyl group of powder particles and carboxylic groups of polyacrylic acid by, which decreases the working time. Also, it may react with -Si-O- $\mathrm{Si}-$ units at the surface of the glass particles, leading to the formation of $-\mathrm{Si}-\mathrm{OH}$ groups. ${ }^{20,21}$ Also the presence of amine and hydroxyl groups on the chitosan is the most probable site for the accommodation of the additional water. The presence of hydroxyl and carboxyl 
groups in polycarboxylic acid and their resultant polymer make them more hydrophilic and more prone to water sorption consequently water solubility. ${ }^{22}$

On adding small amounts of chitosan into glass ionomer cement there were improved mechanical properties. If the interfacial tension between each component whether high, or weak, the mechanical properties of the material were considered to be poor. ${ }^{23}$ Therefore, addition of additive like chitosan forms a network with polyacrylic acid, the inorganic particles which decrease the interfacial tension within the glass ionomer cement components, which helps the mechanical properties of the material to improve, ${ }^{24}$ this phenomena explains how the polymeric network binds strongly around the inorganic filler. ${ }^{25}$

\section{CONCLUSIONS}

The force generated during normal occlusion and para functional movements increase the marginal deterioration and causes microleakage at the margins of Class $\mathrm{V}$ restorations. The results of this study show that the experimental group containing chitosan - modified glass ionomer cements showed less microleakage, but further clinical evaluation for other physical and biological properties of the experimental cement should be considered under occlusal loading.

Data sharing statement provided by the authors is available with the full text of this article at jemds.com.

Financial or other competing interests: None.

Disclosure forms provided by the authors are available with the full text of this article at jemds.com.

\section{REFERENCES}

[1] Rencken JE, Wolke J. Clinical and SEM assessment of ART high-viscosity glass-ionomer sealants after 8 to 13 years in 4 teeth. J Dent 2010;38(1):59-64.

[2] Gupta SK, Gupta J, Saraswathi V, et al. Comparative evaluation of microleakage in class $\mathrm{V}$ cavities using various glass ionomer cements: an in vitro study. J Interdiscip Dentistry 2012;2(3):164-9.

[3] Tikhonov VE, Stepnova EA, Babak VG, et al. Bactericidal and antifungal activities of a low molecular weight chitosan and its $\mathrm{N}$-/2(3)-(dodec-2-enyl) succinoyl/derivatives. Carbohydr Polym 2006;64(1):66-72.

[4] Gyulbenkiyan E, Gusiyska A, Vassileva R, et al. Scanning electron microscopic evaluation of the sealer/dentine interface of two sealers using two protocols of irrigation. JIMAB 2020;26(1):2887-91.

[5] Eleih-Ali-Komi D, Hamblin MR. Chitin and chitosan: production and application of versatile biomedical nanomaterials. Int J Adv Res (Indore) 2016;4(3):411-27.

[6] Farag RK, Mohamed RR. Synthesis and characterization of carboxymethyl chitosan nanogels for swelling studies and antimicrobial activity. Molecules 2013;18(1):190-203.

[7] Shariatinia Z. Carboxymethyl chitosan: properties and biomedical applications. Int J Biol Macromol 2018;120(Pt B):1406-19.
[8] Imai $\mathrm{T}$, Watanabe $\mathrm{T}$, Yui $\mathrm{T}$, et al. The directionality of chitin biosynthesis: a revisit. Biochem J 2003;374(Pt 3):755-60.

[9] Azuma K, Izumi R, Osaki T, et al. Chitin, chitosan and its derivatives for wound healing: old and new materials. J Funct Biomater 2015;6(1):104-42.

[10] Martău GA, Mihai M, Vodnar DC. The use of chitosan, alginate and pectin in the biomedical and food sectorbiocompatibility, bioadhesiveness and biodegradability. Polymers (Basel) 2019;11(11):1837.

[11] Zabrovsky A, Beyth N, Pietrokovski Y, et al. Biocompatibility and functionality of dental biometerials. Dental Materials 2017:245-36.

[12] Ito M. In vitro properties of a chitosan-bonded hydroxyapatite bone-filling paste. Biomaterials 1991;12(1):41-5.

[13] Park SS, Kim SG, Lim SC, et al. Osteogenic activity of the mixture of chitosan and particulate dentin. J Biomed Mater Res A 2008;87(3):618-23.

[14] Hayashi Y, Ohara N, Ganno T, et al. Chitosan-containing gum chewing accelerates antibacterial effect with an increase in salivary secretion. J Dent 2007;35(11):871-4.

[15] Shin SY, Park HN, Kim KH, et al. Biological evaluation of chitosan nanofiber membrane for guided bone regeneration. J Periodontol 2005;76(10):1778-84.

[16] Ibrahim MA, Priyadarshini BM, Neo J, et al. Characterization of chitosan $/ \mathrm{TiO}_{2}$ nano-powder modified glass-ionomer cement for restorative dental applications. J Esthet Restor Dent 2017;29(2):146-56.

[17] Rao DJG, Balasubramanian NN, William BJ, et al. Clinical evaluation of chitin and chitosan in the management of wounds. Tamilnadu J Vet Animal Sci 2007;3:160-3.

[18] Goldstein RE, Lamba S, Lawson NC, et al. Microleakage around class $\mathrm{V}$ composite restorations after ultrasonic scaling and sonic tooth brushing around their margin. J Esthet Restor Dent 2017;29(1):41-8.

[19] Chandrasekhar V, Reddy LP, Prakash TJ, et al. Spectrophotometric and colorimetric evaluation of staining of the light cured composite after exposure with different intensities of light curing units. J Conserv Dent 2011;14(4):391-4.

[20] Pillai CKS, Paul W, Sharma CP. Chitin and chitosan polymers: chemistry, solubility and fiber formation. Progress in Polymer Science 2009;34(7):641-78.

[21] Sidhu SK, Nicholson JW. A review of glass-ionomer cements for clinical dentistry. J Funct Biomater 2016;7(3):16.

[22] Ibrahim MA, Neo J, Esguerra RJ, et al. Characterization of antibacterial and adhesion properties of chitosanmodified glass ionomer cement. J Biomater Appl 2015;30(4):409-19.

[23] Malacarne J, Carvalho RM, Mario F, et al. Water sorption/solubility of dental adhesive resins. Dent Mater 2006;22(10):973-80.

[24] Petri DFS, Donega J, Benassi AM, et al. Preliminary study on chitosan modified glass ionomer restoratives. Dent Mater 2007;23(8):1004-10.

[25] Elbahrawy EM, Rahim RAA. Effect of addition of chitosan on water sorption, solubility and microhardness of glass ionomer cement. Tanta Dent J 2017;14(3):164-8. 